\title{
Place and Position Learning in Honeybees (Apis mellifera)
}

\author{
Brigitte Huber, P. A. Couvillon, and M. E. Bitterman
}

\begin{abstract}
Foraging honeybees (Apis mellifera) were trained individually to choose between 2 identical targets set close together on a large table in a heterogeneous surround. Discrimination was facilitated by the introduction of a small object that was nearer to 1 target than the other. It was also facilitated by the introduction of a longer object or a curved shield that was not differentially placed with respect to the targets but designed to encourage a fixed orientation to them. The results support a distinction between place learning and position learning in honeybees.
\end{abstract}

When a honeybee is trained to choose between two targets that differ in some integral property, such as color, the locations of the targets must be interchanged to demonstrate discrimination of color apart from location. Discrimination of location, which may be evident in a preference displayed at the outset of training or established by differential reinforcement (Couvillon, Klosterhalfen, \& Bitterman, 1983; Klosterhalfen, Fischer, \& Bitterman, 1978 ), is interesting because location is not an integral property of a target but may be given in relation either to its surround (place learning) or to the orientation of the animal (position learning).

Place and position learning were first clearly distinguished in some early experiments with rats (Blodgett \& McCutchan, 1947; Tolman, Ritchie, \& Kalish, 1946). Trained on an elevated T maze whose situation in a visually heterogeneous environment is changed from trial to trial, rats can learn readily to go always to a fixed place, whether a left or a right turn at the choice point is required to take them there on any given trial. They also can learn-more readily in a homogeneous environment than in a heterogeneous one (Restle, 1957) - to turn always to the left or always to the right at the choice point, although the same turn takes them to different places on different trials. Learning always to turn left or right at a choice point has traditionally been characterized as response learning rather than position learning on the assumption that the motor system is uniquely involved. That assumption is clearly expressed by Leonard and McNaughton (1990) in a recent essay on the neurobiology of spatial representation in rats: "The response strategy," they wrote, "employs a specific sequence of motor acts, largely independently of the distribution of sensory cues, to attain a goal of navigation" (p. 375). Our own preference is for the more neutral term, position learning, because persuasive evidence for the motor interpretation is

Brigitte Huber, P. A. Couvillon, and M. E. Bitterman, Békésy Laboratory of Neurobiology, University of Hawaii.

This research was supported by Grant IBN-9308132 from the National Science Foundation.

We thank Peter D. Balsam for helpful comments.

Correspondence concerning this article should be addressed to M. E. Bitterman, Békésy Laboratory of Neurobiology, 1993 East-West Road, Honolulu, Hawaii 96822 . Electronic mail may be sent to jeffb@ahi.pbrc.hawaii.edu. lacking. It seems at least equally plausible that choice may be based on "position in visual space," as Wehner (1981, p. 536) suggested in his analysis of pattern discrimination in honeybees.

Place learning in honeybees has been studied in landmark experiments of several different kinds. One useful technique is to feed foragers on a target near a distinctive object or array of objects, and then to record their searching behavior in the absence of the target after some objects have been altered, repositioned, or removed (e.g., Cartwright \& Collett, 1982; Cheng, Collett, Pickhard, \& Wehner, 1987; Collett \& Kelber, 1988; Wehner, 1981). Another technique is to train foragers to choose between two identical targets, with nearby objects to indicate which of the targets contains food on any trial (e.g., Couvillon \& Bitterman, 1992). Evidence of position learning in honeybees comes from experiments with confined foragers limited to walking in a maze (Weiss, 1954) or in a Y-shaped discrimination chamber (Sigurdson, 1981). In the present experiments with free-flying subjects, place and position learning are contrasted in the same foraging situation.

\section{Experiment 1}

The subjects of this experiment were trained to discriminate between two identical targets at different locations on a homogeneous surface in a differentiated surround.

\section{Method}

Subjects. The subjects were 36 foraging honeybees (Apis mellifera), all experimentally naive, from our own hives in the vicinity of the laboratory. They were recruited and trained individually.

Procedure. The experimental situation is sketched in Figure 1. The targets were displayed on the $90 \times 180 \mathrm{~cm}$ wooden top of a table set outdoors at one end of the south wall of our laboratory building. The table was about $120 \mathrm{~cm}$ from the wall under the eaves of the building in a two-sided enclosure that was differentiated by shaded windows, metal shelving, a rack of timing and recording equipment, air conditioning units, and shrubbery. Animals approached from the south by flying around the east wall of the enclosure. The targets were petri dishes $(5.5 \mathrm{~cm}$ in diameter), with their covers sprayed with gray paint.

The pretraining procedure in this and all subsequent experiments was as follows: An animal was selected at random from a group of 


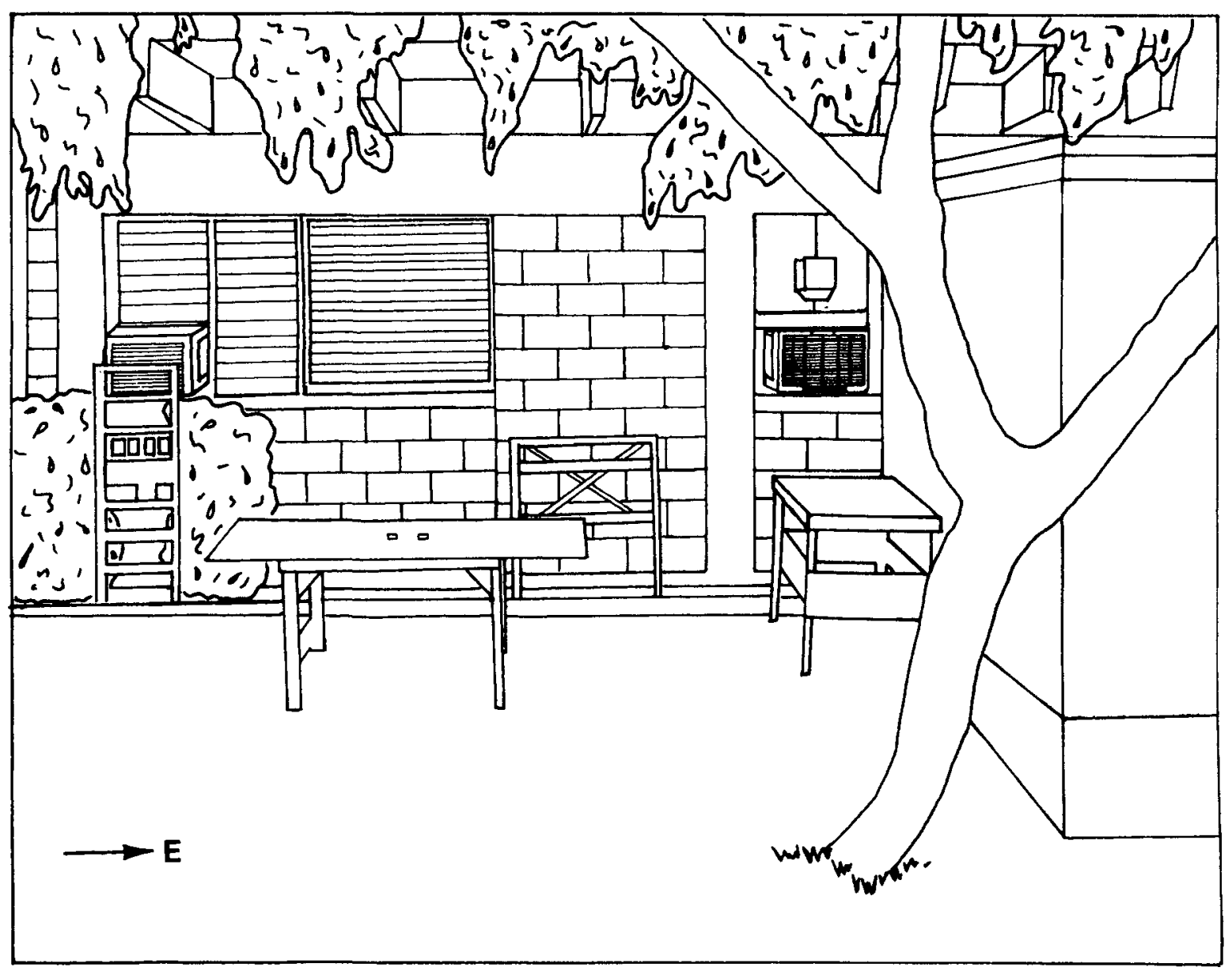

Figure 1. A sketch of the experimental situation, which shows the two targets, $10 \mathrm{~cm}$ apart, centered on the $90 \times 180 \mathrm{~cm}$ top of the training table.

foragers at a feeding station equipped with a jar of $10 \%-12 \%$ sucrose solution that was out of sight of the training situation. The animal was captured in a match box, carried to the table, and placed at a large drop of $50 \%$ sucrose solution on a single target situated at the center of the table. There the animal was permitted to feed to repletion (during which time it was marked with an identifying spot of colored lacquer) and then to fly back to the hive. Typically, the animal returned to the table in a few minutes and continued to shuttle back and forth between the hive and the table as long as sucrose was available there. If it did not return to the table after its first placement, it was picked up at the feeding station (where it usually could be found) and placed again on the target. Choice training began on the visit after the animal's first return to the table of its own accord and continued for a total of 16 training visits.

One group of animals (Group $40 ; n=12$ ) was trained with a pair of targets placed $40 \mathrm{~cm}$ apart (edge-to-edge) at the center of the table in an east-west arrangement, as shown in Figure 1. One of the targets $(\mathrm{S}+)$ contained a $100-\mu$ l drop of $50 \%$ sucrose solution from which feeding to repletion was permitted, and the other target (S-) contained a $100-\mu$ l drop of water, unacceptable to the animals and distinguishable from the sucrose only by taste. If $S-$ was chosen on any trial, an error was recorded, and correction was permitted; the subject fed to repletion on each trial, which ended with the subject's return to the hive. A second group of animals (Group 10;n=12) was trained as was Group 40, except that the targets were only $10 \mathrm{~cm}$ apart. For a third group of animals (Group $10 \mathrm{~V} ; n=12$ ), the targets also were $10 \mathrm{~cm}$ apart, but the placement of the pair during training was varied (without rotation-the eastwest orientation was maintained) over the four quadrants of the table in balanced quasi-random fashion, four trials in each quadrant. Six of the 12 animals in each group were trained with the east target as $\mathbf{S}+$ and the rest, with the west target as $\mathbf{S}+$. The targets used on each visit were washed and exchanged for others in a set of identical targets after the visit in order to randomize extraneous stimuli.

On the visit after the 16 th training visit, there was a 10 -min extinction test in which each target now contained a $100-\mu l$ drop of water. For Groups 40 and 10 , the targets were situated as they were in training; for Group $10 \mathrm{~V}$, the targets were at the center of the table, exactly as for Group 10. When an animal encountered water on one of the targets, it left the target, then returned to the same target or went to the other, left again, and returned again to one of the targets (sometimes only briefly, with no attempt to drink), and so forth; the interval between successive responses increased as the test continued. All actual contacts with each target, however brief, during a 10 -min period were recorded by the experimenter, who pressed one of two hand-held switches that 
activated counters programmed to print stored frequencies at $30-\mathrm{s}$ intervals. Work with each animal was terminated at the conclusion of the extinction test.

\section{Results}

In Figure 2 (which includes selected acquisition data from this and from subsequent experiments), the performance of Groups 10 and 40 is plotted in terms of the mean number of erroneous choices in successive blocks of four acquisition trials. Group 40 made significantly fewer errors than was expected by chance ( $p<.05$, binomial expansion), but Group 10 did not $(p>.05)$. In Figure 3, the performance of the two groups in the extinction test is plotted in terms of the mean cumulative number of responses to each target in successive 30 -s intervals. Group 40 significantly preferred $\mathrm{S}+, F(1,11)=11.18, p<.01$, but Group $10 \mathrm{did}$ not $(F<1)$. These results can be understood on the assumption that the $40-\mathrm{cm}$ separation was sufficient to permit place learning (differentiation of the targets in terms of features of the larger surround adjacent to them), although the $10-\mathrm{cm}$ separation was not.

The mean number of errors made by Group $10 \mathrm{~V}$ did not decline significantly over blocks of acquisition trials $(p>.05$ ), but in extinction (Figure 3), the animals showed a significant preference for $S+, F(1,11)=9.48, p=.01$. The extinction measure, which is based on many more responses than the acquisition measure, is, of course, the more reliable of the two, and it may be expected also to be

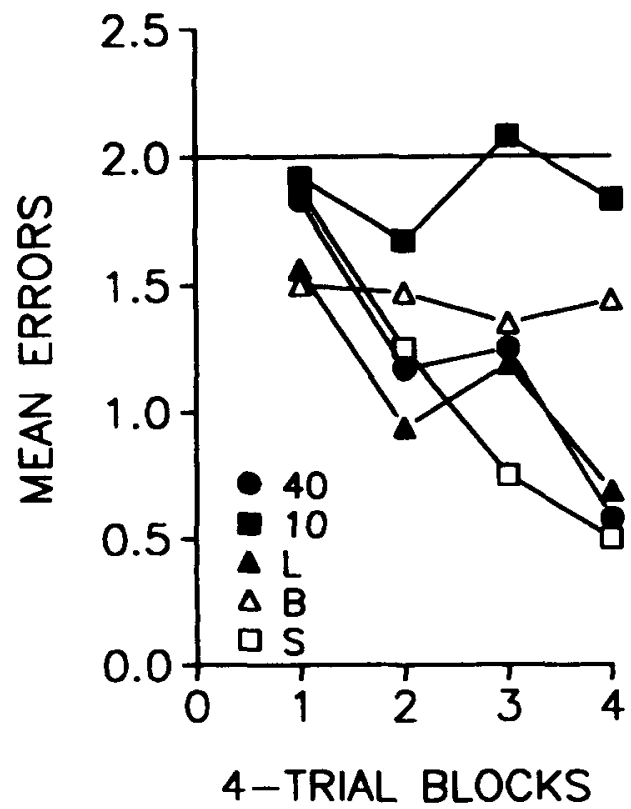

Figure 2. The course of acquisition under five conditions in which the position of the pair of targets remained the same. $40=$ Group $40 ; 10=$ Group 10; $\mathrm{L}=$ Groups LP and $\mathrm{LN}$ (both trained with the landmark); $\mathrm{B}=$ Groups $\mathrm{BN}, \mathrm{BS}, \mathrm{BD}$, and $\mathrm{B} 0$ (all trained with the bar); $S=$ Group $S$ (trained with the shield).

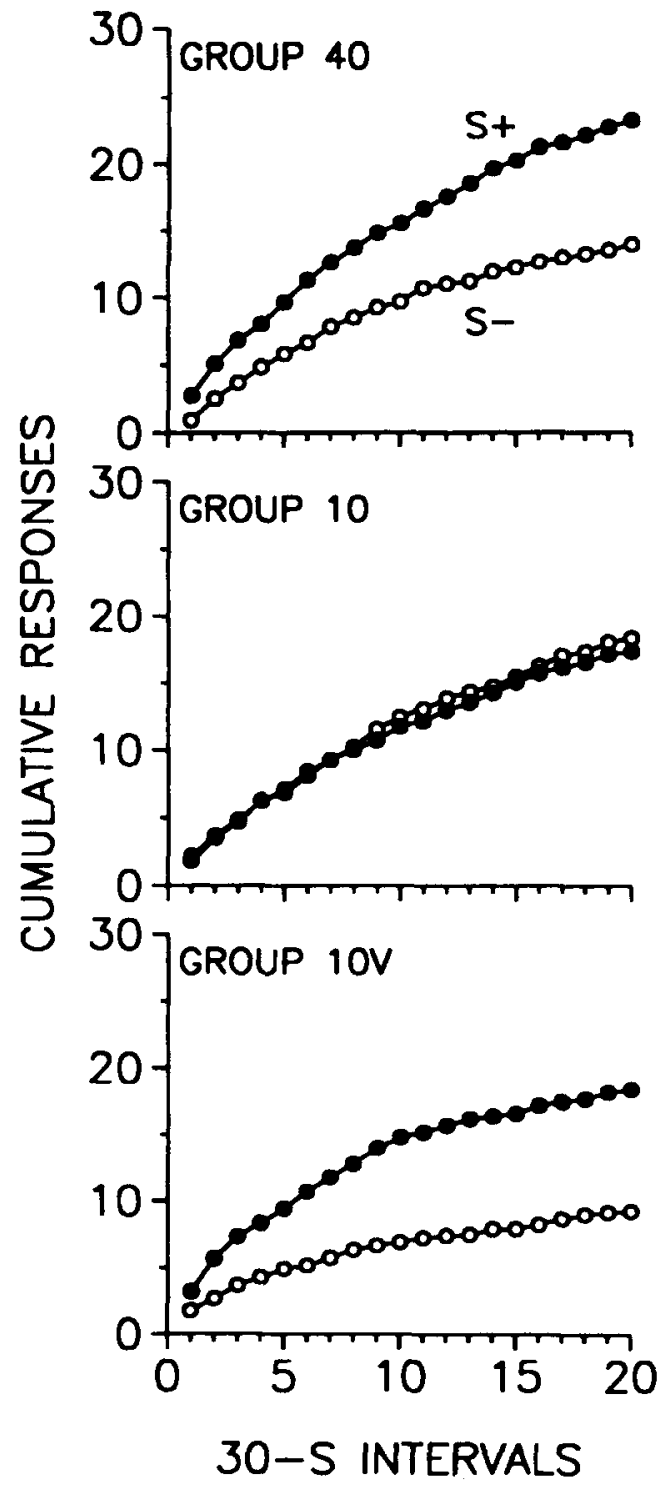

Figure 3. Performance in the extinction test of the three groups trained with the targets alone in Experiment 1.

the more sensitive, given that it is made at the end of acquisition. The preference of Group $10 \mathrm{~V}$ for $\mathrm{S}+$ is unlikely to reflect place learning because the location of the pair of targets was varied widely in training and was never, as in the test, at the center of the table. A better explanation may be that the Group 10V animals adopted a common orientation to the targets that served as the basis for position learning. The common orientation was perhaps more likely to develop in Group $10 \mathrm{~V}$ than in Group 10 because continued inspection of the situation on departure was promoted by the variation in training locations; Lehrer (1991) found that variation in training conditions increases the frequency with which departing foragers turn back and look at the feeding situation. It is possible also, of course, that the variation facilitated the development of a common orienta- 
tion by promoting inspection on approach. A detailed videographic analysis of behavior both on arrival and departure will be instructive.

\section{Experiment 2}

In Experiment 1, 10-cm separation of two targets presented always at the center of the table was assumed to permit neither place nor position learning. In Experiment 2, the separation was the same, but a small object was introduced that was nearer one of the targets than the other and might serve, therefore, as a local landmark. The arrangement was expected to permit place learning.

\section{Method}

Subjects. The subjects were 32 foraging honeybees, all experimentally naive, from our own hives. They were recruited and trained individually.

Procedure. Two groups of animals were trained with two targets $10 \mathrm{~cm}$ apart (edge-to-edge) at the center of the table in the east-west arrangement and, as shown in the top portion of Figure 4 , with a landmark $5 \mathrm{~cm}$ away from one of the targets-east for half the animals in each group and west for the rest. The landmark was a blue wooden block, $4 \mathrm{~cm}$ wide $\times 4 \mathrm{~cm}$ high $\times 9 \mathrm{~cm}$ long, with its long dimension in the north-south orientation. For Group LP $(n=8)$, the target nearer the landmark was $\mathrm{S}+$. For Group LN $(n=8)$, the target nearer the landmark was $S-$. In extinction the configuration was the same as in training.

Two other groups of 8 animals each-Groups LPR and LNR-were trained as were the first two, except that the location of the target and landmark configuration on the table was changed from trial to trial, and it was rotated in $90^{\circ}$ steps as well, all in balanced quasi-random sequence. On one trial in each quadrant, the configuration was as shown in Figure 4, with the targets in the east-west orientation and the landmark to the west; on a second trial in each quadrant, the targets were in a north-south orientation with the landmark to the north; on a third trial in each quadrant, the targets were in an east-west orientation with the landmark to the east; and on a fourth trial in each quadrant, the targets were in a north-south orientation with the landmark to the south. The extinction test was given with centered targets in the east-west arrangement, with the landmark to the east for half the animals in each group and to the west for the rest.

\section{Results}

In acquisition, Groups LP and LN developed a significant preference for $\mathrm{S}+(p=.01)$; their pooled performance, which was much the same (and much like that of Group 40), is plotted in Figure 2. Their performance in extinction, which also was much the same, is pooled in Figure 5. Analysis of variance yields a significant preference for $\mathrm{S}+, F(1,14)=22.27, p<.001$, but neither a significant group effect $(F<1)$ nor a significant Group $\times$ Stimulus interaction $(F<1)$. It is clear that the introduction of a distinguishing landmark compensated for reduced separation of the targets that impaired the performance of Group 10 in relation to that of Group 40 . It is clear also

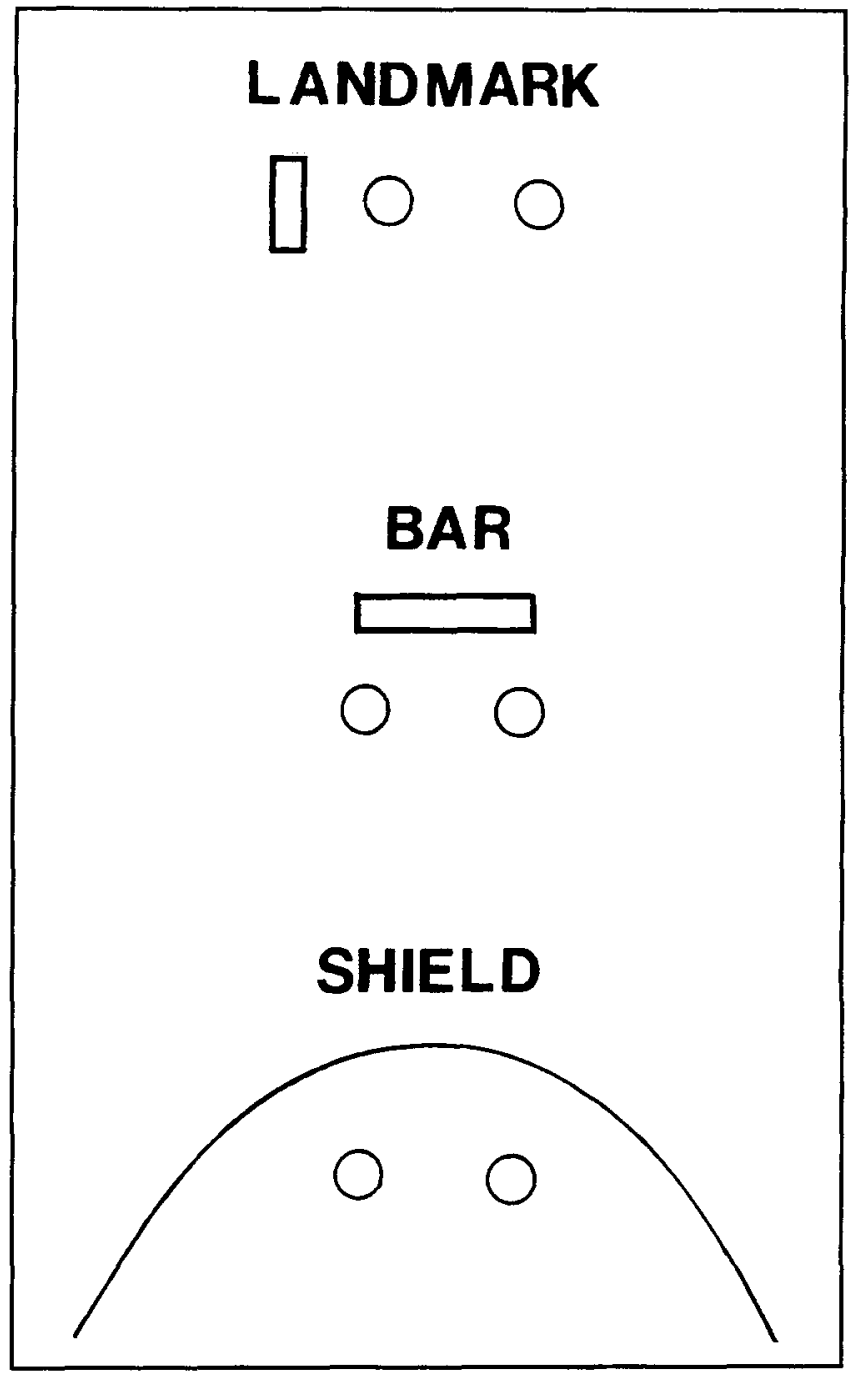

Figure 4. The target and landmark configuration of Experiment 2 , the target and bar configuration of Experiment 3, and the target and shield configuration of Experiment 4.

that the landmark could serve equally well as a positive or a negative signal. Equal performance in landmarkpositive and landmark-negative training has previously been reported by Couvillon and Bitterman (1992).

The performance of Groups LPR and LNR, for which the landmark and target configurations were displaced and rotated from trial to trial, did not improve significantly in acquisition $(p>.05)$. Their performance in extinction (pooled in Figure 5) did, however, show a significant preference for $S+, F(1,14)=45.33, p<.0001$, with neither a significant group effect $(F<1)$ nor a significant Group $\times$ Stimulus interaction $(F<1)$. It is clear from the extinction results that displacement and rotation did not prevent the development of a preference; the critical determinant of performance, apparently, was proximity to the landmark, which defined the place at which sucrose was to be found. 


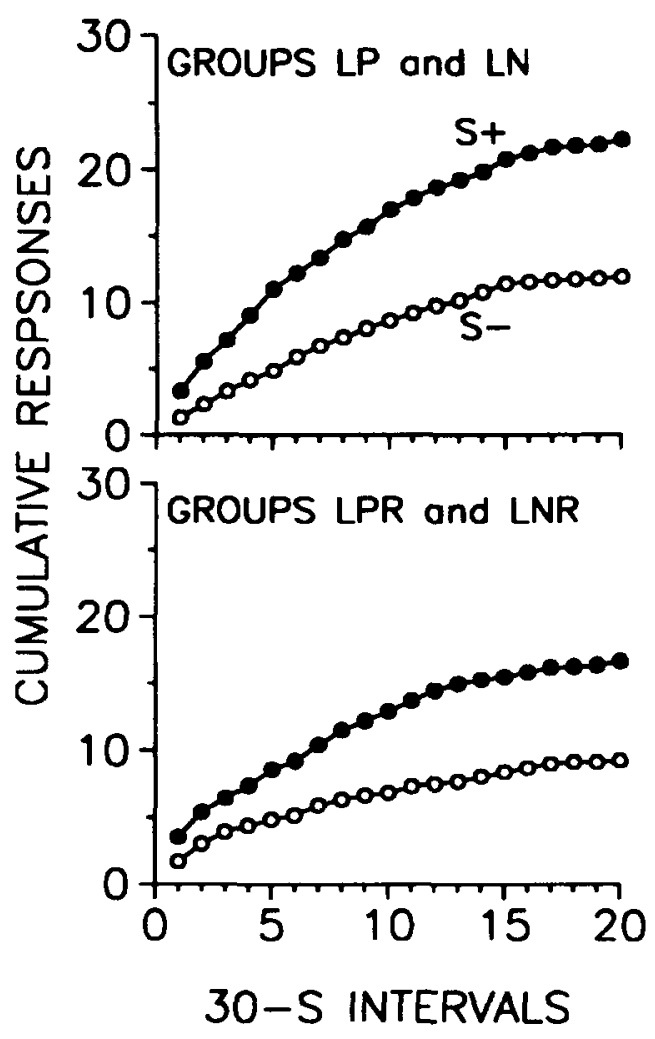

Figure 5. Performance in the extinction test of the groups trained with the landmark in Experiment 2.

\section{Experiment 3}

In Experiment 3, we looked again for differential response to targets separated by $10 \mathrm{~cm}$ but with a local object - a bar-that was placed symmetrically with respect to the targets. The bar was not a landmark in the special sense that the block used in Experiment 2 could be called a landmark, because the bar did not in itself distinguish between the targets, although it probably did help to define their location as a pair. The bar was introduced on the intuition that it might encourage a fixed orientation to the targets and thus facilitate position learning.

\section{Method}

Subjects. The subjects were 40 foraging honeybees, all experimentally naive, from our own hives. They were recruited and trained individually.

Procedure. The target and bar configuration is diagrammed in Figure 4 . The bar was a blue block, $18 \times 4 \times 4 \mathrm{~cm}$. For animals in Group BN $(n=8)$, the bar was situated $5 \mathrm{~cm}$ north of the two targets, which were at the center of the table $(10 \mathrm{~cm}$ apart in the east-west arrangement), both in training and in testing. For animals in Group BS $(n=8)$, the bar was $5 \mathrm{~cm}$ south of the targets both in training and testing. For half the animals in each group, the east target was $\mathrm{S}+$; for the rest, the west target was $\mathrm{S}+$.
Two other groups were trained as were Groups BN and BS, except that the bar position was balanced within rather than between groups-north for half the animals in each group and south for the rest. Again the east target was $S+$ for half the animals in each group, and the west target for the rest. For the animals of Group BD $(n=8)$, the position of the bar was different in extinction than in training-south for animals trained with the bar to the north, and north for animals trained with the bar to the south. For the animals of Group B0 $(n=8)$, the bar was absent in extinction.

A fifth group of animals (Group BR; $n=8$ ) was trained with the location of the target and bar configuration changed from trial to trial-four trials in each of the four quadrants of the table-and rotated in $90^{\circ}$ steps as well (all in balanced, quasi-random sequence). On one trial in each quadrant, the configuration was as shown in Figure 4, with the bar to the north; on a second trial, the bar was to the south; and on a third and a fourth trial, the orientation of the targets was north-south with the bar either to the east or to the west. As the target and bar configuration is diagrammed in Figure 4, the left target was $S+$ for half the animals and the right target, for the rest. In extinction the targets were at the center of the table in the east-west arrangement with the bar to the north.

\section{Results}

The pooled acquisition results for Groups BN, BS, BD, and B0, each of which was trained with a centered and unrotated configuration, are plotted in Figure 2 . The number of errors was significantly less than chance $(p<.0001)$, but there was not the same systematic improvement in performance in this case as in the landmark case. In extinction, Groups BN and BS, whose pooled performance is plotted in Figure 6, both showed a significant preference for $S+, F(1$, 14) $=37.62, p<.0001$, with neither a significant group effect $(F<1)$ nor a significant Group $\times$ Stimulus interaction $(F<1)$. Whereas the two targets were equidistant from the bar, the bar could not have functioned as a simple landmark. Our hypothesis is that the bar in some way facilitated orientation to the targets, making position learning possible. The extinction results for the other three bar groups also are plotted in Figure 6. The fact that none of them showed a significant preference for $S+(F<1$ in each case) suggests that the orientation assumed to permit position learning in Groups BN and BS was jointly controlled by the bar and by some more remote feature of the context. The results for Groups BD and B0 show the continuing importance of the bar; displacing it, or removing it entirely, impaired discrimination. The results for Group BR, whose preference for $\mathbf{S}+$ was not different from chance either in training or in the extinction test, show that a fixed relation between the target and bar configuration and the larger context was essential.

\section{Experiment 4}

In Experiment 4, a salient local surround-a tall, white, curved shield-was substituted for the bar. The shield was intended to exert more powerful control of the direction of approach to the targets and at the same time to minimize the 


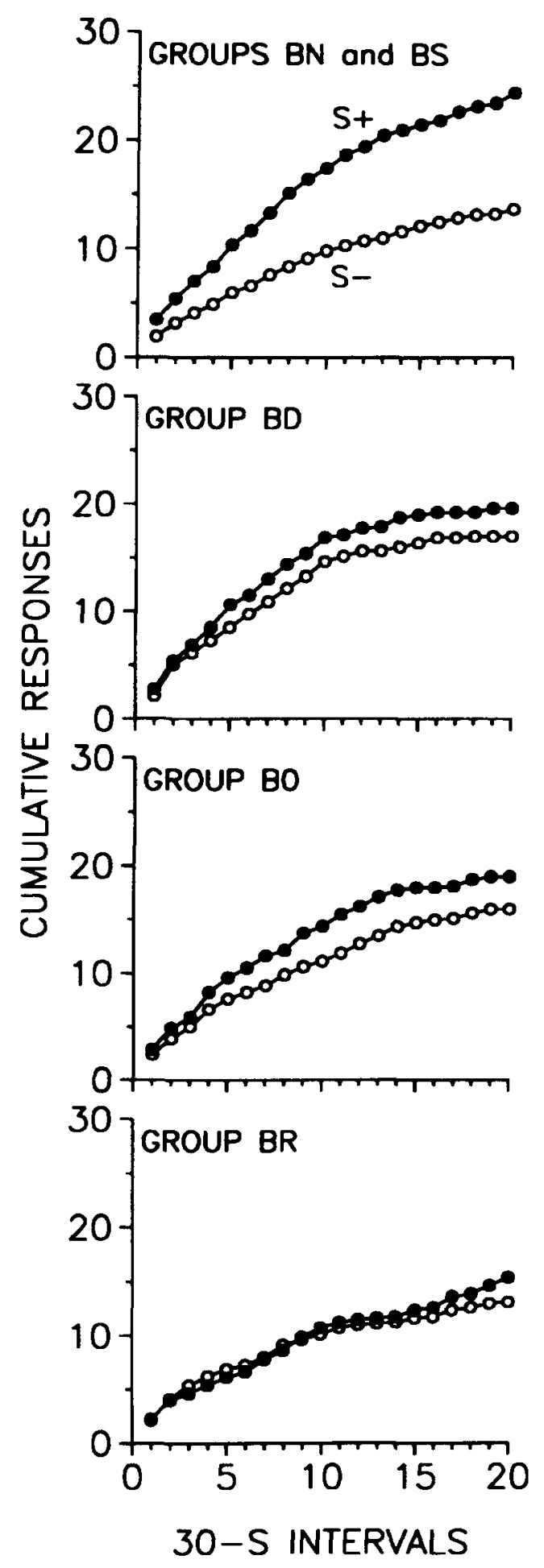

Figure 6. Performance in the extinction test of the groups trained with the bar in Experiment 3.

influence of the larger environment at the point of choice. Our hope was that it might provide a standard set of conditions for studying the relation between position learning and learning about other target properties.

\section{Method}

Subjects. The subjects were 8 foraging honeybees, all experimentally naive, from our own hives. They were recruited and trained individually.

Procedure. For a single group of 8 animals, designated as Group $S$, a white curved shield, $50 \mathrm{~cm}$ high and $71 \mathrm{~cm}$ wide, was set to the north of the two targets. Both in training and in testing, the targets were centered on the table in the east-west arrangement, $10 \mathrm{~cm}$ apart and $10 \mathrm{~cm}$ from the shield. The geometry is shown in Figure 4. The east target was $S+$ for half the animals, and the west target was $S+$ for the rest.

\section{Results}

The course of learning in Group $S$ is shown in Figure 2 . The number of errors was significantly less than chance $(p<.0001)$, and the plot shows the same pronounced improvement as was found in Group 40 and in the landmark groups (Groups LP and LN) for which the placement of the targets was unchanged during training. In the extinction test the animals showed a strong and significant preference for $\mathrm{S}+, F(1,7)=92.56, p<.0001$. The extinction curves are plotted in Figure 7.

\section{Discussion}

In Experiment 1, the locations of the two identical targets alone at the center of the table were clearly discriminated when they were separated by $40 \mathrm{~cm}$ (Group 40) but not when they were separated by only $10 \mathrm{~cm}$ (Group 10). The simplest explanation is that the animals of Group 40 were responding on the basis of contextual stimuli, which were not sufficiently different for targets that were closer together; that is, there was place learning in Group 40 but not in Group 10. Direct evidence of place learning was found in Experiment 2, in which the addition of a local landmark that was closer to one of the targets than to the other made

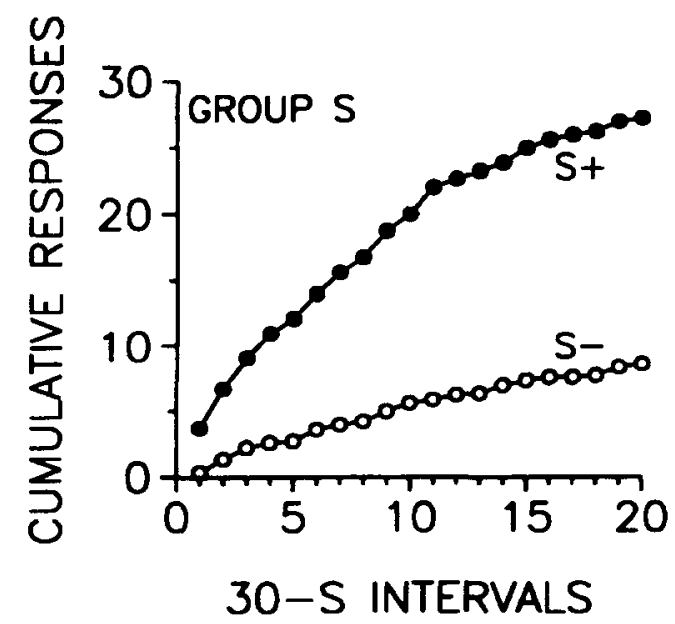

Figure 7. Performance in the extinction test of the group trained with the shield in Experiment 4. 
discrimination possible even with a separation of only 10 $\mathrm{cm}$. Rotation of the target and landmark configuration during training to some extent impaired acquisition as measured in terms of errors but did not prevent the development of a clear preference for the rewarded location as evidenced by the subsequent extinction test; that is, control by the local landmark transcended control by features of the surroundings, as Cheng et al. (1987) also reported, although remote features sometimes may play a dominant role (Cartwright \& Collett, 1982), as a function, one must suppose, of their relative salience. How landmarks work remains to be determined, of course.

Our first indication of location learning that is not place learning was provided in Experiment 1 by Group 10V, for which the placement of the pair of targets, separated by 10 $\mathrm{cm}$, was systematically varied (without rotation) in the course of training. Preference for $S+$ in the extinction test suggested the development of a common orientation to the targets, which then could be differentiated in terms of position. Experiment 3 showed that discrimination could be facilitated also by the use of a local object-the bar-that did not in itself distinguish the two targets but seemed in conjunction with the surroundings to promote the development of a fixed orientation to the targets. The popular snapshot metaphor (Cartwright \& Collett, 1982) does not help us to understand why the bar and landmark configurations ought to be differentially susceptible to rotation. In Experiment 4, with the bar replaced by a high curved shield, a substantial preference for $\mathrm{S}+$ appeared both in training and extinction; like the bar, the shield did not in itself distinguish the two targets but can be supposed to have exerted more powerful control of the direction of approach to them. As has already been noted, the idea that honeybees discriminate position in the visual field was suggested some years ago by Wehner (1981), who stressed the importance for honeybees and other flying insects of "fixating objects of interest" (p. 393) and speculated on the way in which direction of approach is determined.

Our own interest in the problem has been stimulated by the results of experiments on the discrimination of what are best now referred to as color-location compounds (Couvillon et al., 1983; Klosterhalfen et al., 1978). Although the term position rather than location was used in the reports of those experiments, the discrimination may as well have been of place, because the targets were presented in locally differentiated surroundings. (The distinction between position and place learning was not considered at the time.) The color-location results are intriguing because they differ from those of color-odor experiments; for example, dimensional transfer is found with color-location compounds but not with color-odor compounds (Klosterhalfen et al., 1978). The differences can perhaps be understood on the assumption that color and odor are integral target properties and that position functions like one, but place (if that is what actually was studied) does not. It will be interesting to do experiments of the same kind both under the place-learning conditions of Experiment 2 and the position-learning conditions of Experiment 4.

\section{References}

Blodgett, H. C., \& McCutchan, K. (1947). Place versus response learning in the simple T-maze. Journal of Experimental Psychology, 37, 412-422.

Cartwright, B. A., \& Collett, T. S. (1982). How honey bees use landmarks to guide their return to a food source. Nature, 295, 560-564.

Cheng, K., Collett, T. S., Pickhard, A., \& Wehner, R. (1987). The use of visual landmarks by honeybees: Bees weight landmarks according to their distance from the goal. Journal of Comparative Physiology A, 161, 469-475.

Collett, T. S., \& Kelber, A. (1988). The retrieval of visuo-spatial memories by honeybees. Journal of Comparative Physiology A, $163,145-150$.

Couvillon, P. A., \& Bitterman, M. E. (1992). Landmark learning by honeybees. Journal of Insect Behavior, 5, 123-129.

Couvillon, P. A., Klosterhalfen, S., \& Bitterman, M. E. (1983). Analysis of overshadowing in honeybees. Journal of Comparative Psychology, 97, 154-166.

Klosterhalfen. S., Fischer, W., \& Bitterman, M. E. (1978). Modification of attention in honeybees. Science, 201, 1241-1243.

Lehrer, M. (1991). Bees which turn back and look. Naturwissenschaften, 78, 274-276.

Leonard, B., \& McNaughton, B. L. (1990). Spatial representation in the rat: Conceptual, behavioral, and neurophysiological perspectives. In R. P. Kestner \& D. S. Olton (Eds.), Neurobiology of comparative cognition (pp. 363-422). Hillsdale, $\mathrm{NJ}$ : Erlbaum.

Restle, F. (1957). Discrimination of cues in mazes: A resolution of the "place vs. response" question. Psychological Review, 64, 217-228

Sigurdson, J. E. (1981). Automated discrete-trials techniques of appetitive conditioning in honeybees. Behavior Research Methods \& Instrumentation, 13, 1-10.

Tolman, E. C., Ritchie, B. F., \& Kalish, D. (1946). Studies in spatial learning: II. Place learning versus response learning. Journal of Experimental Psychology, 36, 221-229.

Wehner, R. (1981). Spatial vision in arthropods. In H. Autrum (Ed.), Comparative physiology and evolution of vision in invertebrates: C. Invertebrate visual centers and behavior II (pp. 287-616). Berlin: Springer.

Weiss, K. (1954). Der Lernvorgang bei einfachen Labyrinthdressuren von Bienen und Wespen [The learning of simple mazes by bees and wasps]. Zeitschrift für Vergleichende Physiologie, 36, 9-20.

Received August 17, 1993

Revision received October 4, 1993

Accepted October 6, 1993 